\title{
Launching a Laboratory Testing Process Quality Improvement Toolkit: From the Shared Networks of Colorado Ambulatory Practices and Partners (SNOCAP)
}

\author{
Douglas Fernald, MA, Mika Hamer, MPH, Kathy James, PhD, Brandon Tutt, MA, \\ and David West, PhD
}

Introduction: Family medicine and internal medicine physicians order diagnostic laboratory tests for nearly one-third of patient encounters in an average week, yet among medical errors in primary care, an estimated $15 \%$ to $54 \%$ are attributed to laboratory testing processes. From a practice improvement perspective, we (1) describe the need for laboratory testing process quality improvements from the perspective of primary care practices, and (2) describe the approaches and resources needed to implement laboratory testing process quality improvements in practice.

Methods: We applied practice observations, process mapping, and interviews with primary care practices in the Shared Networks of Colorado Ambulatory Practices and Partners (SNOCAP)-affiliated practice-based research networks that field-tested in 2013 a laboratory testing process improvement toolkit.

Results: From the data collected in each of the 22 participating practices, common testing quality issues included, but were not limited to, 3 main testing process steps: laboratory test preparation, test tracking, and patient notification. Three overarching qualitative themes emerged: practices readily acknowledge multiple laboratory testing process problems; practices know that they need help addressing the issues; and practices face challenges with finding patient-centered solutions compatible with practice priorities and available resources.

Conclusion: While practices were able to get started with guidance and a toolkit to improve laboratory testing processes, most did not seem able to achieve their quality improvement aims unassisted. Providing specific guidance tools with practice facilitation or other rapid-cycle quality improvement support may be an effective approach to improve common laboratory testing issues in primary care. (J Am Board Fam Med 2015;28:576-583.)

Keywords: Diagnostic Tests, Practice-based Research, Primary Health Care, Quality Improvement

Diagnostic laboratory testing is common in the primary care setting. Family medicine and internal medicine physicians order diagnostic laboratory tests for nearly one-third of patient encounters in

This article was externally peer reviewed.

Submitted 14 January 2015; revised 5 June 2015; accepted 15 June 2015.

From the Department of Family Medicine, University of Colorado School of Medicine, Aurora (DF, MH, KJ, DW); and the Colorado Health Outcomes Program, University of Colorado School of Medicine, Aurora (MH, BT, DW).

Funding: The research described in this article was funded by the Centers for Disease Control and Prevention, Division of Laboratory Programs, Standards, and Services, via cooperative agreement no. U47CI000832.

Conflict of interest: none declared. an average week. ${ }^{1}$ Among medical errors in primary care, an estimated $15 \%$ to $54 \%$ are attributed to laboratory testing processes. ${ }^{2}$ Laboratory testing errors can occur at any point in the testing process, including the preanalytic steps (eg, test selection, test ordering, and specimen collection) and postanalytic steps (eg, reporting results, interpreting results, and notifying patients). Causes of laboratory testing errors are broadly attributable to process failures, delays, communication gaps, errors in

Corresponding author: Douglas Fernald, MA, Department of Family Medicine, University of Colorado School of Medicine, 12631 E. 17th Ave., MS F496, Aurora, CO, 80045 (E-mail: doug.fernald@ucdenver.edu). 
judgment and cognition, influence of language, practice culture, and lack of patient centeredness. ${ }^{3}$ Critical handoffs occur between the clinician and laboratory, and while failures in this space rarely have catastrophic consequences, patients may more commonly experience emotional distress or delayed diagnosis and practices may suffer financial loss. ${ }^{4}$

With the roll out of meaningful use standards by the Centers for Medicare and Medicaid Services and provisions of the Affordable Care Act that are moving the health care system from a fee-for-service model to a pay-for-performance model, there is significant incentive for practices to improve the quality of their laboratory testing processes. Intrinsic demand for these types of quality improvement (QI) efforts at the primary care practice level may be leveraged to earn economic incentives such as those available through extrinsic mechanisms like the Meaningful Use Program offered by Centers for Medicare and Medicaid Services, and other programs supported by the Affordable Care Act.

This study is the culmination of the final phase of a 3-part project funded by the Centers for Disease Control and Prevention. In phase 1 of the project, the team conducted a review of the literature to assist in the development of quality indicators that could improve pre- and postanalytic phases of the laboratory testing process for primary care practices. Identified root causes of errors fell into 7 major themes: process failures, delays, communication gaps, errors in judgment and cognition, influence of minorities/language, practice culture, and lack of patient centeredness. ${ }^{3}$ In phase 2 of the project, the team administered a survey to assess perceptions of laboratory hand-off gaps in primary care practices in Colorado to better understand the perceived gaps in these processes and as a precursor to the initiation of process improvement activities. Identified commonalities in test tracking and patient notification challenges warranted the development of a laboratory testing toolkit to facilitate QI in pre- and postanalytic testing processes. ${ }^{5}$

In the third and final phase of this project, the objective was to describe the process of implementing a laboratory process QI toolkit in primary care practice settings. Using data collected before and after the development and implementation of the Toolkit, we (1) describe the need for laboratory testing process QIs from the perspective of primary care practices, and (2) describe the approaches and resources needed to implement laboratory testing process QIs in practice.

\section{Methods \\ Context}

In 2010, the Centers for Disease Control and Prevention funded the University of Colorado Health Outcomes Program to design QI initiatives that would bridge current primary care-laboratory medicine hand-off gaps. To assess perceptions regarding gaps in primary care pre- and postanalytic laboratory testing processes, a survey of personnel among member practices in the Shared Networks of Colorado Ambulatory Practices and Partners (SNOCAP) practice-based research networks identified perceived gaps in laboratory testing processes by key role within the primary care practice, and it identified specific lab testing processes as targets for in-depth study before initiating process improvement. ${ }^{5}$

\section{Toolkit Development}

Based on previous study results, ${ }^{3,5}$ a laboratory testing process toolkit was developed and piloted in 6 primary care practices. The pilot consisted of a "real-world" test of the Toolkit to select, design, and execute an intervention designed to improve a key phase of the laboratory testing process, such as test preparation and ordering, test tracking, results follow-up, or patient notification. Pilot practices provided comments on both the improvement process and the Toolkit content. Practice observations, process mapping, and interviews were conducted to understand how the Toolkit should be revised. Based on the pilot testing and established practice improvement principles, ${ }^{6,7}$ a revised Toolkit was produced in January 2013 for use in the large-scale roll-out and field test of the Toolkit. The Toolkit included brief, step-wise guidance to assist practices in (1) getting started, (2) aligning practice priorities, (3) preparing for change, (4) assessing current lab testing processes, (5) planning an improvement activity, (6) collecting baseline data, (7) implementing the improvement activity, and (8) assessing the outcomes of the change (Table 1).

\section{Practice Recruitment}

We recruited ambulatory care practices from SNOCAP practice-based research networks affiliated with the University of Colorado Department 
Table 1. Summary of Laboratory Testing Process Improvement Toolkit Contents

\begin{tabular}{|c|c|}
\hline Section & Content \\
\hline Background on Laboratory Testing and Patient Safety & $\begin{array}{l}\text { - Summary of the scope of laboratory testing in primary care } \\
\text { - Key points from the literature about laboratory testing, patient safety, } \\
\text { and medical errors } \\
\text { - Examples of primary care laboratory testing process improvements }\end{array}$ \\
\hline Step 1: How Do We Get Started? & $\begin{array}{l}\text { - Quick self-assessment of laboratory issues in practice (checklist) } \\
\text { - Planning for a team to lead the improvement effort (checklist) }\end{array}$ \\
\hline Step 2: Priority Alignment & $\begin{array}{l}\text { - Tips for aligning laboratory process improvement efforts with practice } \\
\text { priorities } \\
\text { - Priority alignment assessment (worksheet) }\end{array}$ \\
\hline Step 3: Making It Real \& Preparing for Change & $\begin{array}{l}\text { - Tips for successful launch of the improvement effort } \\
\text { - "Preflight" communication reminders (checklist) }\end{array}$ \\
\hline $\begin{array}{l}\text { Step 4: What Is Your Current Process for Laboratory } \\
\text { Testing? }\end{array}$ & $\begin{array}{l}\text { - Overview of how to map current laboratory testing processes } \\
\text { - Process mapping guidance (step-by-step instructions and example) } \\
\text { - Steps to review the process map (checklist) }\end{array}$ \\
\hline Step 5: How Are You Going to Fix the Problem? & $\begin{array}{l}\text { - Step-by-step planning guide for developing and implementing an } \\
\text { improvement plan (checklist) } \\
\text { - Improvement plan template (worksheet) }\end{array}$ \\
\hline $\begin{array}{l}\text { Step 6: How Do You Know If You Made a } \\
\text { Difference? }\end{array}$ & $\begin{array}{l}\text { - Importance of (and tips for) selecting metrics to evaluate the success } \\
\text { of the improvement plan } \\
\text { - Measurement selection planning tool (checklist and worksheet) }\end{array}$ \\
\hline Step 7: Make the Change! & - Planning tips for executing the improvement effort (worksheet) \\
\hline Step 8: Did We Improve Our Lab Testing Process? & $\begin{array}{l}\text { - Guidance on reviewing data and planning for next steps } \\
\text { - Steps to review and use improvement data (checklist) }\end{array}$ \\
\hline
\end{tabular}

of Family Medicine. A recruitment E-mail with information on study aims was sent to practice managers or medical directors. Practices that did not respond received a follow-up E-mail 4 to 6 weeks after the initial invitation. A payment of $\$ 1200$ was offered to each practice for study-related activities. This study was reviewed for human subjects protections and was approved by the Colorado Multiple Institutional Review Board.

\section{Practice Intervention}

Beginning in January 2013, for each enrolled practice, the study team conducted an initial site visit to introduce the Toolkit and study requirements, and to conduct an initial process mapping and observation activity. Resulting process maps were offered to practices to help guide their decision processes, but they were not required to participate in the study. Each practice was provided with the laboratory testing Toolkit and minimal guidance on the use of the Toolkit, with the expectation that they would use it in whatever way they determined was most practical to help identify and implement a QI process around laboratory testing processes. Practices then were given approximately 6 weeks to select an improvement activity focus, design a brief intervention, test the intervention, and collect qual- ity data (a common time frame for rapid QI projects).

\section{Data Sources and Analysis}

A follow-up interview was conducted to gather additional data on the QI activities and on the usability of the Toolkit. Field notes from observation visits, process maps, Toolkit feedback forms, and interview data were organized into meta-matrices for analysis. ${ }^{8}$ Data were reviewed iteratively, focusing first on the step-wise process practices followed to reflect, plan, design, and implement a QI activity for their laboratory testing processes. From the initial analysis phases, emergent themes were documented and presented to the research team for further assessment and reexamination of the data. The main themes that emerged are presented in the Results, using case vignettes to illustrate key needs and implementation strategies to address quality gaps in laboratory testing processes.

\section{Results}

Of 31 practices recruited in an implementation field test of the Toolkit, 24 agreed to participate, and a final group of 22 actively participated. Actively participating practices included a range of primary care settings, including small and medium- 
Table 2. Laboratory Testing Process Improvement Activities Selected by Practices and Stage of Completion by Study End

\begin{tabular}{|c|c|c|c|}
\hline Clinic ID & Testing Phase & QI Activity Focus & $\begin{array}{l}\text { Toolkit Stage } \\
\text { Completed }\end{array}$ \\
\hline & Preanalytic & & \\
\hline 21 & Test preparation & $\begin{array}{l}\text { Improve documentation of patient notification preferences and documentation } \\
\text { of scheduled lab visits. }\end{array}$ & Step 6 \\
\hline 22 & Test selection & $\begin{array}{l}\text { Improve the identification of patients requiring repeat MRSA testing to reduce } \\
\text { the number of patients remaining on an MRSA protocol list. }\end{array}$ & Step 6 \\
\hline 1 & Test ordering & $\begin{array}{l}\text { Reduce the number of lab tests rejected because of incorrect coding in } \\
\text { electronic medical record. }\end{array}$ & Step 3 \\
\hline 2 & & $\begin{array}{l}\text { Reduce the number of lab tests rejected because of incorrect coding in } \\
\text { electronic medical record. }\end{array}$ & Step 3 \\
\hline 5 & & Reduce the number of incomplete laboratory orders sent from clinic. & Step 7 \\
\hline 19 & & Reduce the number of incomplete laboratory orders sent from clinic. & Step 1 \\
\hline 3 & $\begin{array}{l}\text { Specimen } \\
\text { collection }\end{array}$ & Improve rate of completed specimen collection by using on-site phlebotomy. & Step 8 \\
\hline 9 & & Improve rate of completed specimen collection by using on-site phlebotomy. & Step 6 \\
\hline \multirow[t]{2}{*}{11} & & Improve rate of completed specimen collection by using on-site phlebotomy. & Step 7 \\
\hline & Postanalytic & & \\
\hline 7 & Test result tracking & $\begin{array}{l}\text { Reduce the number of lab tests ordered that are not reconciled with results } \\
\text { that can be found in the medical record. }\end{array}$ & Step 5 \\
\hline 8 & & $\begin{array}{l}\text { Improve patient notification processes and documentation to reduce calls from } \\
\text { patients requesting test results. }\end{array}$ & Step 5 \\
\hline 12 & & $\begin{array}{l}\text { Standardize the order tracking system to reduce number of missing labs } \\
\text { requiring patient notification. }\end{array}$ & Step 8 \\
\hline 16 & & $\begin{array}{l}\text { Improve the lab tracking process by implementing a review process for an } \\
\text { EHR-generated report of outstanding lab tests. }\end{array}$ & Step 5 \\
\hline 17 & & $\begin{array}{l}\text { Improve the lab tracking process by implementing a review process for an } \\
\text { EHR-generated report of outstanding lab tests. }\end{array}$ & Step 5 \\
\hline 20 & & $\begin{array}{l}\text { Improve the lab tracking process by implementing a review process for an } \\
\text { EHR-generated report of outstanding lab tests. }\end{array}$ & Step 8 \\
\hline 10 & Patient notification & $\begin{array}{l}\text { Standardize patient notification processes to ensure } 100 \% \text { patient notification } \\
\text { attempts of all laboratory test results. }\end{array}$ & Step 7 \\
\hline 13 & & $\begin{array}{l}\text { Improve the timeliness of provider review of lab results to reduce number of } \\
\text { patient calls. }\end{array}$ & Step 7 \\
\hline 14 & & $\begin{array}{l}\text { Standardize the patient notification processes to ensure } 100 \% \text { patient } \\
\text { notification attempts of all laboratory test results. }\end{array}$ & Step 7 \\
\hline 15 & & $\begin{array}{l}\text { Improve the timeliness of provider review of lab results to reduce number of } \\
\text { patient calls. }\end{array}$ & Step 8 \\
\hline 18 & & $\begin{array}{l}\text { Improve the patient notification processes and documentation to reduce calls } \\
\text { from patients requesting test results. }\end{array}$ & Step 3 \\
\hline 4 & Did not select & Did not select & Step 4 \\
\hline 6 & Did not start & Did not start & Did not start \\
\hline
\end{tabular}

EHR, electronic health record; MRSA, methicillin-resistant Staphylococcus aureus; QI, quality improvement.

sized federally qualified health centers, residency programs, and smaller, privately owned clinics. There were 11 rural practices and 11 urban practices, with wide geographic dispersion across the state of Colorado. Overall, 20 of the 22 practices were able to initiate a laboratory testing improvement process using the Toolkit as a guide, though progress through all steps provided in the Toolkit was uneven; just 4 practices were able to complete their improvement activities within the 6-week pe- riod allotted. Many continued their effort after the end of the study period (Table 2). Two practices did not successfully start an improvement activity.

Comments about the Toolkit were consistently positive in terms of the contents, tools, and guidance, noting that the Toolkit was straightforward, the organization of the Toolkit was helpful, and it provided good talking points to get staff and providers "on the same page." Several practices pointed out specifically that the process mapping 
exercise was very helpful: "The exercise of creating a process map was very, very helpful. It forced us to think critically and about the lab process that were we intervening on. It forced us to ask questions about all the details to answer 'who did what' in the process. Creating a process map [is] essential!"

The suggestions for additional improvements included adding tips for dealing with perceived process failures, providing additional suggestions for sharing positive improvement experiences, and eliminating "hard stops" in the tools so practices do not feel like they cannot move forward if they answer "no" to a question in the Toolkit. Although the entire Toolkit (including appendices) is just 22 pages, staff and providers underscored the importance of keeping language brief and simple so they can quickly digest the contents.

From the follow-up data collected from each of the 22 participating practices, 3 overarching qualitative themes emerged, which we detail below: practices know they have problems; practices know they need help addressing those problems; and practices faced challenges in finding/implementing patient-centered solutions compatible with practice priorities and available resources.

\section{We Know We Have Problems}

Initially, from the participating practices we learned of an overwhelming need for process improvement for laboratory testing processes. When prompted, practices could easily describe multiple lab testing problems, though few had executed successful efforts to mediate these issues. In many cases it seemed that practices were overwhelmed by the number of issues with their lab testing processes, expressing that their pressing issues "had existed for years." In one case, a practice described that, "It was determined that a process already existed to track the labs, but had never been implemented." In another case multiple problems were identified:

1. "Lab orders are not in the patient chart. Patient checks in for lab draw at the Red Pod, and the provider has not entered lab orders for the patient."

2. "When the provider does not sign the future orders, and the patient comes in, the lab techs are unable to complete the "processing lab orders' piece that is required by us for IT and the billing side of [the electronic medical record]."
3. "Providers [are] not completing their orders on the [electronic medical record] order screen."

4. "The lab staff met to think about the problems associated with the lab orders not being completed. We discussed the issue with the medical director and she agreed that it is a problem and agreed to support a plan to change."

Despite being able to easily enumerate the issues, it seemed that the specifics of problems and process improvement had been discussed infrequently at the practice level, and that consensus among clinicians and practice staff needed to be reached. One clinic team member commented that, "we got started by acknowledging that the problem existed."

Practice Vignette 1
Test ordering: missed blood draws
Practice X is a small practice in rural, south-central Colorado.
For this federally qualified health center, most laboratory tests
are sent to two commercial reference laboratories. The practice
is EHR-enabled and most lab test results are automatically
returned by the laboratories to the ordering clinician's inbox.A
review of their EHR data indicated that too many patients were
not completing blood work ordered by the provider. They met as
a team and reviewed the current workflows and staffing. They
determined that they had sufficient staff and space to set up a
phlebotomy room to test their intervention. They also redesigned
the workflow to have the provider walk the patient to the
in-clinic draw station after ordering tests. The QI team reported
that: "Reviewing the original and updated [EHR] report showed
a vast improvement of compliance of blood collection." Upon
further assessment of he intervention they identified a need for
more trained medical staff to perform phlebotomy to further
improve rates of blood draws at the time of the patient visit.

The introduction of the Toolkit, followed by the practices' first interdisciplinary team meetings, resulted in a rapid acknowledgment of ongoing problems, concerns, as well as an ability to quickly and easily secure support from the leadership to develop and act on plans to improve laboratory testing processes. It was clear that awareness of these problems predated the intervention designed to address them. In addition, through the process mapping activity, 3 overarching types of self-identified problems emerged. 
First, practices identified miscommunication and role confusion with regard to lab test ordering and patient notification. Practices described generation of paper requisitions either from an electronic health record [EHR] or paper order that was sometimes the role of the medical assistant or nurse or, alternatively, the back office staff. In some cases lab results were returned to a clinician other than the ordering provider or they were not returned at all.

Second, practices described insufficient pre- and postanalytic lab testing processes. This was most commonly expressed as no formal, written process to retrieve outside labs, to know if a specimen was not analyzed, to know if a result was not sent, to reconcile patients who do not return, and/or to recover specimens not received by the lab.

Third, process mapping identified duplication of efforts resulting in workflow inefficiency. In one case phlebotomists were frequently performing all orders in a patient file, not recognizing that some were future orders, resulting in a need to resample the patient at a future visit. In another case data were being entered 3 times. This practice asked, "Is a simpler entry approach possible?"

Practice teams acknowledged that they needed to make the time to meet as a team to tackle the lab testing issues that had plagued them in the past, and they found the time that they took to do this to be worthwhile and that it yielded results. A representative of 1 practice team told us that:

"We started off with the initial meeting with everyone to determine the issues and solutions to these problems. We set a 1-week trial period to put all the ideas in place. After that week, we met again to see if things were working better, what did not work or if there was a need for a change. We did another follow up meeting a month after that. The staff was really excited about this project so they were all very willing to give input and to make these changes."

\section{We Know We Need Help}

While practices understood the need to make specific changes within their own organization, they also came to realize that some solutions required external support (eg, assistance with a kick-off meeting, assistance with process mapping, or reference lab assistance). Without exception, after the introduction of the Toolkit to practices, project representatives were invited to conduct a kick-off meeting with the entire interdisciplinary practice team. The meeting was identified by practices as serving 2 purposes: (1) to introduce the Toolkit and generate discussion of specific lab testing problems and their impact on patient safety and patient care, and (2) to provide assistance to map processes that were identified by practices as worrisome or problematic.

\section{Practice Vignette 2 \\ Test tracking: unreliable test tracking reports}

Practice $\mathrm{Y}$ is a large family medicine residency practice in Colorado with its clinic located in a building on the sponsoring hospital's campus. They routinely participate in QI projects as part of their resident training and participation in quality improvement programs. Because of staffing shortages, faculty physicians were responsible for leading and executing the QI effort. The residency program's sponsoring hospital contracted with a third-party reference laboratory that provided most of the routine laboratory testing needs. Through a brief survey of staff and providers about their top priorities for laboratory testing issues they agreed that reducing the time spent by "doctor of the day" reviewing and adjudicating all incoming laboratory test results would reduce overall paper flow through this EHR-enabled office. Pre-intervention manual tracking results showed that the "doctor of the day" had to reconcile 268 tests in the one-week baseline data collection period (mean of 34 tests/day). A new workflow required a medical assistant to compare available paper lab requisitions with "labs queued" in the EHR. If the paper requisition had both a "queued" lab and a complete result in the EHR, the paper was discarded. Any remaining "orphaned" paper requisitions were reviewed by the "doctor of the day" for adjudication and follow up. This routine was conducted daily. After the reports were fixed in the EHR, post-intervention results showed that the "doctor of the day" had to reconcile just 10 tests in the one-week follow-up data collection period (mean of 1 test/day).

The process of introducing the Toolkit most often took the form of discussing and prioritizing the ongoing problems with pre- and postanalysis lab processes that the practice was experiencing. The interdisciplinary team, however, often had problems prioritizing the relative importance of these easily identifiable (and common) problems, based on the impact on patient care and office workflows, as well as the feasibility to effectively address the problems. Practice teams requested and received assistance from project representatives to 
talk through their issues, reflect on possible solutions, and ultimately choose a first area of focus for process improvement. Despite having an acute awareness of the ongoing problems that they identified, practices consistently required this external presence and facilitation to choose a topic.

Once a topic or area of focus was chosen, practices also requested assistance to facilitate the development of a process map for use in accurately portraying current processes before setting out to improve them. Using a large whiteboard, practice teams were usually able to effectively visualize and map the process or processes that had been chosen by the team. Having multidisciplinary participation in these efforts was paramount to ensuring an accurate portrayal of processes, including the identification of multiple parallel processes that were in place to accomplish the same end that were being used within the same practice. Once completed, drawings and notes were taken away by the project representatives, and formal process maps were developed using commercial software, along with accompanying narratives and change strategy plans.

In all, these efforts required approximately 20 person-hours of effort (including research assistant and investigator time) to hold the meetings and develop these materials for each practice. While not an unreasonable amount of time and effort, it was beyond the capacity of practice members and practice staff to take time away from their assigned duties to complete these facilitation tasks, which proved to be critical to moving ahead with any meaningful improvement activities. It also was clear that the presence of the project representatives served to legitimate the entire process improvement activity for the entire practice team.

\section{Developing Process Improvement Interventions}

The issues identified by practices included both pre- and postanalytic phases (Table 2), and improvements focused on test ordering, test tracking, and patient notification. In most clinics more than 1 high-priority laboratory testing process gap was identified. Proposed solutions involved information system queries and reports, manual tracking systems, provider reminders, staffing changes, workflow changes, and improvements to existing protocols related to ordering, tracking, and patient notification. In several clinics paper and EHR data systems were used side by side to improve reconciliation procedures for identifying and acting on

\section{Practice Vignette 3}

Patient Notification: Slow patient notification and too many patient calls

Practice $\mathrm{Z}$ is a small practice in rural, south-central Colorado. This federally qualified health center hasis EHR-enabled and most lab test results are automatically returned by the laboratories tothe ordering clinician's inbox, but the nursing staff and front desk staff experienced a high volume of calls from patients asking for their test results. The nursing staff further noted that they are often waiting for patient follow-up instructions from providers as they have not yet reviewed the test results. After reviewing the process, they identified part of the problem being providers who had received lab testresults in their EHR "inbox," but were not reviewing them and deciding on action in a timely manner. They chose to fix the problem by encouraging providers to review their inbox daily and communicate more quickly with the nursing staff to direct their follow-up with patients. The improvement process yielded better teamwork and communication between the providers and their staff, and, ultimately, fewer patient calls asking for their lab test results.

labs that were ordered but "not resulted" or labs that were "resulted" but not reviewed and sent off for patient notification or patient follow-up. Involvement of practice staff and clinicians varied by practice and depended on existing QI infrastructure, methods used, and personnel available during the study period.

We were interested in understanding how the Toolkit would perform in primary care practices with relatively little assistance from the project team to more closely approximate a "real world" test of the Toolkit. Largely left alone to work through the Toolkit and QI processes over a period of about 6 to 8 weeks, just 4 practices that participated were able to complete an entire QI cycle to fully test and evaluate an improvement to their laboratory testing processes and report data. Among those practices that did not complete a full QI cycle, the Toolkit seemed to help them anticipate and plan specific details about future steps, such as describing specific staffing and workflow changes or describing the metrics to be used to evaluate their intervention. Yet comments from most practices indicated that they had not fully completed their improvements, noting, for example, that they were continuing to collect data (at baseline or after implementation), were planning to implement the process soon, were planning to introduce a new process at an upcoming meeting, or were still developing a plan for an improvement. 


\section{Discussion}

Primary care practices readily identify problems that they are experiencing with both pre- and postanalytic laboratory medicine, and they simultaneously lament that they do not seem to be able to address them adequately. These problems stem from (but are not limited to) 3 main testing process steps: laboratory test preparation, test tracking, and patient notification of results.

The Toolkit was well received by the practices in this study; comments suggested that it provided useful tools and guidance across the recommend steps. Yet our data illustrate that practices wish to address these issues, but they need help to shepherd the activity to a conclusion with measurable, actionable results.

Given the resource constraints and competing priorities, by itself, a toolkit is likely to be insufficient to yield substantive QIs related to laboratory testing processes in primary care clinics. The interventions we assisted the practices to develop were well received by practice personnel but did not systematically result in the consistent establishment or tracking of metrics of success. We did not leave the practices with a high degree of confidence that their efforts would be sustained or expanded in any systematic way. Our assumption is that without quantifiable improvements, the value proposition for continuing an investment in process improvements in laboratory medicine does not exist. Although practice improvements for laboratory testing are desirable, practices that are supported with practice facilitation may find their change efforts more successful. ${ }^{9}$

This study has several limitations. The study design allowed for a 6-week QI process, which may have been insufficient to allow practices to execute a complete QI improvement cycle and report results on their QI metrics; therefore, we could not fully evaluate how the Toolkit helped in later stages. In addition, without a comparison group, we were unable to assess the relative advantage (or disadvantage) that the Toolkit provided compared with other forms of guidance or no guidance at all. Although the practices in this study represented a range of primary care settings, the sample was limited to Colorado, and the findings may not be generalizable to other states or settings.

The issues practices face in laboratory medicine involve multiple roles and processes within the practice, as well as linkages to organizations outside of the practice (such as clinical reference laboratories). While the Toolkit we developed for this project may have content that, if carefully and systematically used, may indeed result in process and QI, more is needed to provide the incentive to expend the resources required for such change.

The authors give many thanks to Drs. Stephen Raab, Maxwell Smith, and Laurina Williams for their insight and guidance. The authors also acknowledge the practices of BIGHORN, CaReNet, and High Plains Research Network (all practicebased research networks) that participated in the study described in this article. In addition, the authors thank Dr. Mickey Eder for his foundational work and guidance on the laboratory testing processes in primary care.

\section{References}

1. Hickner J, Thompson PJ, Wilkinson T, et al. Primary care physicians' challenges in ordering clinical laboratory tests and interpreting results. J Am Board Fam Med 2014;27:268-74.

2. Hickner JM, Fernald DH, Harris DM, Poon EG, Elder NC, Mold JW. Issues and initiatives in the testing process in primary care physician offices. $\mathrm{Jt}$ Comm J Qual Patient Saf 2005;31:81-9.

3. Smith ML, Raab SS, Fernald DH, et al. Evaluating the connections between primary care practice and clinical laboratory testing: a review of the literature and call for laboratory involvement in the solutions. Arch Pathol Lab Med 2013;137:120-5.

4. Hickner J, Graham DG, Elder NC, et al. Testing process errors and their harms and consequences reported from family medicine practices: a study of the American Academy of Family Physicians National Research Network. Qual Saf Health Care 2008;17:194-200.

5. West DR, James KA, Fernald DH, Zelie C, Smith ML, Raab SS. Laboratory medicine handoff gaps experienced by primary care practices: a report from the Shared Networks of Collaborative Ambulatory Practices and Partners (SNOCAP). J Am Board Fam Med 2014;27:796-803.

6. Langley G, Nolan K, Nolan T, Norman C, Provost L. The improvement guide: a practical approach to enhancing organizational performance. 2 nd ed. San Francisco, CA: Jossey-Bass Publishers; 2009.

7. Solberg LI. Improving medical practice: a conceptual framework. Ann Fam Med 2007;5:251-6.

8. Miles MB, Huberman AM. Qualitative data analysis: an expanded sourcebook. 2nd ed. Thousand Oaks, CA: Sage Publications; 1994.

9. Grumbach K, Bainbridge E, Bodenheimer T. Facilitating improvement in primary care: the promise of practice coaching. New York: Commonwealth Fund; 2012. 